Neurobiol Aging. 2013 February ; 34(2): 451-467. doi:10.1016/j.neurobiolaging.2012.03.011.

\title{
Effects of aging on neural connectivity underlying selective memory for emotional scenes
}

\author{
Jill D. Waring ${ }^{a,}{ }^{*}$, Donna Rose Addis ${ }^{b}$, and Elizabeth A. Kensinger ${ }^{a, c}$

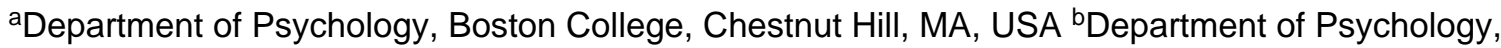 \\ The University of Auckland, Auckland, New Zealand 'Athinoula A. Martinos Center for Biomedical \\ Imaging, Charlestown, MA, USA
}

\begin{abstract}
Older adults show age-related reductions in memory for neutral items within complex visual scenes, but just like young adults, older adults exhibit a memory advantage for emotional items within scenes compared with the background scene information. The present study examined young and older adults' encoding-stage effective connectivity for selective memory of emotional items versus memory for both the emotional item and its background. In a functional magnetic resonance imaging (fMRI) study, participants viewed scenes containing either positive or negative items within neutral backgrounds. Outside the scanner, participants completed a memory test for items and backgrounds. Irrespective of scene content being emotionally positive or negative, older adults had stronger positive connections among frontal regions and from frontal regions to medial temporal lobe structures than did young adults, especially when items and backgrounds were subsequently remembered. These results suggest there are differences between young and older adults' connectivity accompanying the encoding of emotional scenes. Older adults may require more frontal connectivity to encode all elements of a scene rather than just encoding the emotional item.
\end{abstract}

\section{Keywords}

Aging; Selective memory; Emotion; Effective connectivity

\section{Introduction}

Aging is often accompanied by changes in memory for contextual information. Older adults are less likely than young adults to ignore irrelevant contextual details due to inhibitory deficits in flexibly shifting attention (Hasher and Zacks, 1988) or suppressing task-irrelevant contextual information (Gazzaley and D'Esposito, 2007). For instance, older adults are more likely than young adults to falsely recognize scenes that include a familiar background with a novel object (Gutchess et al., 2007). There are also relevant changes in neural activity within the ventral stream: older adults activate visual scene processing regions more than

\footnotetext{
"Corresponding author at: Department of Psychiatry, VA Palo Alto, 3801 Miranda Avenue (MC 151Y), Palo Alto, CA 94304, USA. Tel.: +1 6504935000 ×69414; fax: +1 650858 8926. waringj@ stanford.edu (J.D. Waring).

Supplementary Material

Supplementary data associated with this article can be found, in the online version, at doi:10.1016/j.apmr.2010. 12.027.

Disclosure statement

The authors state there are no actual or potential conflicts of interest.

Participants gave informed consent to participate in a manner approved by the Institutional Review Boards of Massachusetts General Hospital and Boston College.
} 
young adults, but recruit object-processing regions less than young adults (Chee et al., 2006). Together, these behavioral and neural changes suggest that when processing neutral, nonarousing scenes, older adults process contextual information at the expense of memory for relevant item details.

Within emotional scenes, however, there is a robust bias for both young and older adults to process an emotional foreground item at the expense of the background, even when not instructed to do so Kensinger et al., 2005; Reisberg and Heuer, 2004). This effect goes beyond a generic item-processing bias evident for neutral information (Chee et al., 2006; Gutchess et al., 2007; Kensinger et al., 2007a). For this reason, complex visual scenes containing an emotional item are particularly apt for investigating how selective item memory develops. Although there is a wealth of behavioral evidence for selective enhancement in memory for emotional items compared with context (Burke et al., 1992; Kensinger et al., 2006, Kensinger et al., 2007c; Libkuman et al., 2004), it is unclear why it occurs (see discussion by Levine and Edelstein, 2009; Mather and Sutherland, 2011). Even so, there is some evidence to suggest that neural processes during encoding are critical to the development of later selective memory for emotional items. A recent study in young adults confirmed that increased encoding-related activity in the amygdala, hippocampus, fusiform, temporal pole, and inferior frontal gyrus predicted later selective memory for an emotional item from a scene as compared with later remembering both an emotional item as well as its background context (Waring and Kensinger, 2011).

Notably, the enhancement in memory for emotional items is as robust in older adults as in young adults, suggesting that there may be something about the emotional item that "captures" the attention even of the older adults and reduces their typical tendency to process the context of neutral items at the expense of the item (Kensinger et al., 2005; Waring and Kensinger, 2009). In fact, if anything, older adults have a more difficult time switching their encoding resources toward the background when an emotional item is present. Even when instructed to process the whole scene, older adults have more difficulty overcoming selective emotional item memory than do young adults (Kensinger et al., 2005; Kensinger et al., 2007d). One hypothesized mechanism is that inhibitory failures make it harder for older adults to divert their attention away from the emotional item (Kensinger et al., 2005). Thus, although both age groups exhibit selective memory for emotional items over context information, different patterns of neural activity and connectivity may be engaged when young and older adults are able to broaden their focus to encode both the emotional item and also its context; the current study addresses this possibility.

To date, the neuroimaging literature examining the effect of age on item and context memory has focused primarily on neutral scenes. Much of the emerging evidence suggests that there is a general shift in regional activation associated with subsequent memory, from more posterior occipitotemporal regions in young adults to more anterior frontal regions, including dorsolateral and middle frontal areas, in older adults ("posterior to anterior shift in aging" or PASA; Davis et al., 2008; Dennis et al., 2008). It has been hypothesized that these age differences in regional activation patterns reflect compensatory shifts in neural resources, possibly to offset less efficient processing or to engage different strategies (reviewed by Grady, 2008; St. Jacques et al., 2009a). Although older adults have a general impairment in processing contextual information due to disruptions in a dorsolateral prefrontal (dlPFC) cognitive control network, older adults with relatively better memory accuracy additionally engage areas within inferior, middle, and superior gyri to compensate for these cognitive control deficits in memory (Gutchess et al., 2005, 2007). Older adults also have a negative correspondence between activation in inferior frontal gyrus (Brodmann area [BA] 45/46) and parahippocampus, suggesting that prefrontal activation is engaged to compensate for lowered activity in the parahippocampus (Gutchess et al., 2005). 
Studies of neural activation have begun to depict the effects of aging upon the processing and encoding of subsequently remembered images, suggesting that age may not only influence the regions activated, but the interactions between those regions (Dennis et al., 2008; St. Jacques et al., 2010). One way to investigate selective memory in older adults is to examine the changes in neural connectivity underlying age-related differences in encoding processes. Although there has been little research investigating the neural connectivity changes with aging, the extant data reveal that age-related differences in neural connectivity can be revealed even when differences in activity are not (e.g., Dennis et al., 2008; Grady, 2005; Waldinger et al., 2011). Thus, when there are similar patterns of activation supporting memory performance in young and older adults, differences in connectivity between regions may explain age-related differences in memory performance (Grady, 2005). For instance, even when young and older adults show similar regional activation, older adults have shown increased hippocampal to frontal connectivity as well as decreased hippocampal to fusiform connectivity associated with accurate memory for neutral items with their background contexts (Dennis et al., 2008). This change may reflect an increased effect of compensatory activity within anterior regions, yielding a greater influence of executive and controlled processing on subsequent memory, thus offset declining posterior perceptually based processing (Grady, 2005).

With respect to age-related changes in neural activation and connectivity underlying memory for emotional images, there is also evidence of an anterior shift. For example, older adults have been found to show more medial prefrontal and cingulate activation than young adults during successful encoding of positive information, without significant age differences in the pattern of encoding-related activation for negative images (Kensinger and Schacter, 2008). With respect to connectivity, older adults exhibit stronger connectivity between amygdala and dIPFC (BA 45/46 and 47) than young adults during encoding of negative images, which has been hypothesized to be a compensatory mechanism to offset older adults' weakening amygdala-hippocampal connections (St. Jacques et al., 2009b; Murty et al., 2009). Older adults also show stronger amygdala-anterior cingulate connectivity, possibly to facilitate emotion regulation during evaluation of negative images, but at the same time they have decreased connectivity between the amygdala and posterior brain regions reflecting poorer perceptual processing (St. Jacques et al., 2010).

However, in other instances, the connectivity underlying subsequent memory for negative images has not significantly differed between age groups; both age groups have shown strong connectivity among the amygdala, hippocampus, and fusiform underlying successful encoding (Addis et al., 2010). That study also found that young and older adults had differing neural connectivity during the encoding of positive images. Older adults' hippocampal activation was significantly influenced by ventromedial prefrontal (vmPFC) and amygdala projections, while young adults instead had significant thalamic influence upon the hippocampus. Taken together, the evidence has generally shown that older adults have increased functional connectivity between medial temporal and frontal regions to offset weakening connections among medial temporal lobe structures and from medial temporal lobe regions to the fusiform for encoding neutral as well as emotionally valenced images.

One region that may show a somewhat different pattern of age-related change is orbitofrontal cortex (OFC). The OFC modulates the strength of sensory and affective signals to the rest of the brain (Kringelbach and Rolls, 2004), and it has connections throughout the prefrontal cortex and to medial temporal regions, including the amygdala (Cavada et al., 2000). These connections are vital for effective emotional information processing, regulation, and memory (reviewed by Hooker and Knight, 2006), and may be particularly important during the encoding of emotional information because the OFC is disproportionately recruited for encoding of emotionally salient (vs. neutral) visual 
information (Kensinger and Schacter, 2006, 2008; Kensinger et al., 2007a). Although both young and older adults show activation to emotional stimuli within this region (Kensinger and Schacter, 2008), there is some evidence that connectivity between the amygdala and the OFC may be reduced in aging (Moriguchi et al., 2011) and that older adults may be impaired on tasks that are reliant on OFC function (Lamar and Resnick, 2004; Salat et al., 2002).

The purpose of the present study was to compare the neural connectivity for the encoding of emotional scenes between young and older adults to better understand whether there are agerelated changes in connectivity corresponding with selective emotional item memory, or with the ability to encode both the emotional item and the background context. We independently evaluated memory for the two component parts of the visual scene, the emotional item and neutral background; thus, this was not a test of source memory or associative memory. Unlike tests of source memory, participants were not required to remember which scene had been presented with each item. This distinction also made the present study different from a test of associative memory, because here memory was tested for the two components (item and background) independently without reference to their pairing at study. The goal of the present study was to identify the encoding-related activity that led to two different types of memory performance patterns: instances in which the item from a studied scene was endorsed as studied but the background was not, and instances in which both the item and the background from the studied scene were endorsed as studied. This design allowed us to better illustrate age differences in the ties between initial processing and which scene information (item or context) is successfully encoded into memory in order to understand how selective memory develops in young and older adults.

We were interested to find whether older adults would exhibit differences in neural connectivity between regions important for emotional scene memory-namely, the prefrontal cortex (orbitofrontal and dorsolateral), medial temporal regions (amygdala and parahippocampal gyrus), superior parietal lobe, and fusiform gyrus (Kensinger and Schacter, 2006, 2008; Kensinger et al., 2007a). Moreover, by examining memory for positive and for negative stimuli, instead of focusing on only one valence, it was possible to see whether there were similar patterns of connectivity in young and older adults irrespective of valence. We predicted that age-related changes in connectivity underlying memory for both the emotional item and background context may be particularly evident for positive scenes. We hypothesized that when older adults are able to broaden their focus at encoding to remember both the item and context, they would show a compensatory anterior-to-posterior shift in processing, with greater connectivity between the dIPFC, OFC, and medial temporal lobe in older adults than in younger adults. The OFC and amygdala have strong reciprocal connections that are especially relevant for emotion-related learning (reviewed by Kringelbach and Rolls, 2004), however it may be that older adults' selective emotional item encoding is associated with failure to engage these additional prefrontal connections, and the connectivity underlying selective emotional item memory is restricted to medial temporal lobe structures. To further investigate these possibilities, correlational analyses between activation in a priori regions of interest of the amygdala and OFC were used to illustrate the age-related changes in the correspondence between these two regions.

\section{Methods}

\subsection{Participants}

Participants included 19 young adults $(7$ men, 12 women; mean $(M)$ age $=23.67$ years; range, 19-29 years) and 18 older adults ( 7 men, 11 women; age $M=74.4$ years; range, 6783 years). Young adult participants had 14 to 20 years of education $(M=16.7)$ and older adult participants had 12 to 18 years of education $(M=15.9)$. The young adults' neural 
activity (but not connectivity) was reported in a prior study (Waring and Kensinger, 2011). One young adult (female, 24 years old, 18 years of education) was excluded from analyses due to problems obtaining a reliable signal (attributed to scanner spiking). See Table 1 for additional participant characteristics. All were right-handed native English speakers with normal or corrected to normal vision. Participants were screened for history of psychiatric or neurological disorders, or treatment with centrally acting medications. At the time of the study, participants scored within the normal range on standardized measures of depression and anxiety. Participants gave informed consent to participate in a manner approved by the Institutional Review Boards of Massachusetts General Hospital and Boston College. Participants were paid \$25 per hour for participating, prorated to the nearest half hour.

\subsection{Stimuli}

The stimulus set was comprised of 450 complex visual scenes that included a positive, negative, or neutral item placed onto a neutral background. This stimulus set included items and scenes from prior studies (Kensinger et al., 2006, 2007b, 2007c; Waring and Kensinger, 2009) and contained 136 items of each of the 3 emotional valence types (positive, negative, and neutral). Positive and negative items were further subdivided by arousal with a median split (positive median arousal $=2.39$, negative median arousal $=3.86$ ), resulting in 68 negative-high arousal $(M=4.30), 68$ positive high-arousal $(M=2.96), 68$ negative-low arousal $(M=3.24)$, and 68 positive low-arousal $(M=1.85)$ images. Images of emotional and neutral items were placed in the context of neutral backgrounds (backgrounds taken from Kensinger et al., 2007c, and Waring and Kensinger, 2009) to create composite scenes (e.g., a snake by a river; see Fig. 1). The valence of the item placed onto each of the backgrounds was varied across participants (i.e., 1 participant saw a snake [negative item] by a river, another saw a kitten [positive item] by a river; see Fig. 1A) to isolate effects attributed to emotionality of items, and to avoid any confounds related to background of the scenes. Because of a number of constraints imposed on stimulus creation (e.g., plausibility and congruency, described in detail below), it was not possible to fully counterbalance all item and scene pairings; across participants, some items were paired with more than one background, but no participant ever studied an item or a background more than once. Scenes were visually matched by experimenters for size and location of foreground item, and were carefully constructed with strong consideration for the plausibility of each item in the context of the background with which it was paired (e.g., a chipmunk would not be paired with a dining room). Item and background congruency within a scene were assessed by a separate group of 6 raters, showing no significant difference in congruency between the 5 scene types $(F(4,471)=1.14, p=0.34)$, with scenes showing moderate levels of congruency $(M=3.28, S D=1.26$, on a scale of 1-5); participants of the current study were not instructed to evaluate these factors (see Waring and Kensinger, 2009; 2011, for more details on stimuli development).

\subsection{Procedure}

Participants were told that they would see a series of photographic scenes presented inside the scanner, and they were asked to rate each scene according to whether they preferred to approach or retreat from it. Using a 1-3 scale, they chose the corresponding key on a buttonbox indicating their response, where $1=$ approach, $2=$ stay at present location, and $3=$ move away from the scene. This encoding task was specifically chosen because in prior studies it had been shown to reliably elicit selective memory for emotional items in young adults (Kensinger et al., 2007d; Waring and Kensinger, 2009). Scenes were presented for 3 seconds each inside the scanner, followed by a fixation cross (+) for an additional second, so participants had 4 seconds to view each scene and make a response before the program automatically advanced. This was enough time for participants to respond to each scene. Task instructions did not encourage participants to consider speed when making their 
responses and stimuli were all viewed for 3 seconds, regardless of reaction time in responding. Following the approach or retreat decision and a 1-second fixation cross, another fixation cross was presented at variable interstimulus interval (ISI) durations (mean interstimulus interval $=4$ seconds; range $=2-16$ seconds) to provide the jitter required to isolate the hemodynamic response to each stimulus necessary in an event-related design (Dale, 1999). The encoding task was divided into 3 functional runs of 5 minutes each. The studied stimulus set was varied across participants during the scanning session, and scenes were presented in a pseudorandom order for each participant, as determined by the program optseq (written by Doug Greve), to optimize jittering within the functional magnetic resonance imaging (fMRI) environment.

Outside of the scanner and approximately 10 minutes after viewing the scenes, participants took a surprise recognition memory test. Items and backgrounds from the composite scenes were presented independently from one another and intermixed with novel items and backgrounds. The presentation order of test stimuli was varied between participants to minimize artifacts in responses attributable to subject fatigue. Test items included 150 "old" items ( 30 low arousal positive, 30 high arousal positive, 30 low arousal negative, 30 high arousal negative, and 30 neutral) and 150 "old" backgrounds ( 30 had been presented with a low arousal positive item, 30 with a high arousal positive item, 30 with a low arousal negative item, 30 with a high arousal negative item, and 30 with a neutral item), as well as 258 "new" items (152 emotional [ 38 low arousal positive, 38 high arousal positive, 38 low arousal negative, 38 high arousal negative] and 106 neutral) and 150 "new" backgrounds (by definition, all neutral), for a combined total of 708 items and backgrounds. (Memory was tested for a large number of neutral novel items in an attempt to present similar numbers of novel emotional and neutral items at test, and because the novel backgrounds would be all inherently neutral.) The orientation (i.e., horizontal or vertical) of each "old" item presented at test was set to match its orientation in the studied scene; items were never rotated for presentation at retrieval. Participants had 3 seconds to view each test item or background and 7 seconds to select the appropriate key to indicate if it was a component of one of the scenes viewed within the scanner ("old") or had not been seen previously within a scene ("new"). There was a short practice test given before beginning the actual test to assure participants fully understood the meaning of "old" and "new" scene components.

\section{4. fMRI image acquisition}

Images were acquired on a 1.5 T Siemens Avanto magnetic resonance imaging (MRI) scanner. Detailed anatomical data were collected using a multiplanar, rapidly acquired gradient-echo (MP-RAGE) sequence (repetition time $[\mathrm{TR}]=8000 \mathrm{~ms}$, echo time $[\mathrm{TE}]=39$ $\mathrm{ms}$, field of view $(\mathrm{FOV})=200 \mathrm{~mm}$, flip angle $=90^{\circ}$, acquisition matrix $=256 \times 256$ ).

Functional images were acquired using a T2*-weighted echo-planar imaging sequence (TR $=2000 \mathrm{~ms}, \mathrm{TE}=40 \mathrm{~ms}, \mathrm{FOV}=200 \mathrm{~mm}$, flip angle $=90^{\circ}$ ). Twenty-six axial-oblique slices $(64 \times 64$ acquisition matrix, thickness $=3.2 \mathrm{~mm}$, skip factor $=0.64 \mathrm{~mm})$, aligned in a plane along the anterior/posterior commissure line, were acquired in an interleaved fashion (full description of imaging protocol is included in Waring and Kensinger, 2011).

\subsection{Analyses}

2.5.1. Behavioral analyses-Behavioral analyses assessed the effects of age group and scene valence upon memory for scene components. These analyses were restricted only to scenes where the item was successfully remembered; within this set of scenes, instances when the background was forgotten (i.e., selective item memory) were compared to when it was remembered (i.e., subsequent memory for both the item and background; see Fig. 2). In order to match positive and negative scenes for arousal level, only scenes with negative low arousal items and positive high arousal items $(t(118)=1.95, p>0.05)$ were included in 
behavioral and imaging analyses. Description of comparisons including "negative" scene components will refer to only the low arousal negative stimuli, and "positive" scene components will refer to only the high arousal positive stimuli. In this way, the behavioral analysis mirrored the factors assessed in the connectivity models.

2.5.2. Neuroimaging analyses-We used structural equation modeling (SEM) to examine effective connectivity between brain regions during encoding that led to later memory for both item and background versus encoding that led to later selective item memory, as a function of scene valence type, for young and for older adults. SEM can be used to indicate the functional "effect" of one region upon another, and how these influences may differ by condition or participant groups (McIntosh and Gonzalez-Lima, 1994), although it does not imply temporal ordering. Modeling effective connectivity requires developing an anatomical model specifying which brain regions are connected, as well as a functional model of how these regions are interacting with each other for a given condition. The model can then be used to understand whether a given region has a positive or negative influence on each other region (or "node") modeled within the network.

Regions included in the anatomical model were identified using a whole-brain General Linear Model (GLM) analysis in SPM2 (Wellcome Department of Cognitive Neurology). Standard preprocessing was performed on functional data, including slice-timing, rigid body motion correction, normalization to the Montreal Neurological Institute (MNI) template (resampling at $3 \mathrm{~mm}$ cubic voxels), and spatial smoothing (using a $7.6 \mathrm{~mm}$ full-width halfmaximum isotropic Gaussian kernel). Analyses were conducted using a subsequent memory design, modeling data in a subject-specific, fixed-effects, event-related model. An eventrelated analysis was conducted, in which trials were classified into conditions according to recognition accuracy (hit, miss), component type (item, background), and emotion (low arousal positive, high arousal positive, low arousal negative, high arousal negative, neutral), and modeled through convolution with a canonical hemodynamic response function. Participant data from both age groups were then entered into a second-order random effects 1 -sample $t$-test (i.e., collapsing across group) that compared the average neural response to viewing all scene types (irrespective of emotional content or subsequent memory) with baseline at a threshold of $p<0.0001,5$ voxel extent (for results see Supplementary Fig. 1). This whole-brain analysis included all participants so that regions could be defined in an unbiased manner with respect to age and valence. MNI voxel coordinates for local maxima were translated into Talairach coordinate space; these coordinates reflect the most significant voxel within the cluster of activation (Talairach and Tournoux, 1988).

Of the regions identified in this contrast, those that had theoretical relevance to encoding of emotional or detailed visual information (reviewed by Hamann, 2001; Kensinger and Schacter, 2007; LaBar and Cabeza, 2006) were included in the anatomical model: right dlPFC (x, y, z = 50, 13, 27; BA 9), right OFC (34, 36, -15; BA 11), left amygdala ( $-18,-5$, $-20)$, left parahippocampus $(-20,-13,-20)$, left superior parietal lobule $(-24,-64,49$; BA $7)$, and left (-40, -51, -11; BA 37), and right (30, -38, -15; BA 20) fusiform gyri. The connections in the anatomical model were based upon anatomical research in nonhumans and human white matter tractography (Amaral et al., 2003; Patterson and Schmidt, 2003; Swanson and Petrovich, 1998), and considering the relationships between the specified network nodes and direction of pathways (Addis et al., 2007; McIntosh, 1999; Stein et al., 2007).

The functional model was constructed using activity extracted from each node in the model. The percentage signal change in each region was extracted using the MarsBar toolbox in SPM2 (Brett et al., 2002) for all active voxels within a 5-mm sphere centered on the peak voxel. For each participant, the maximum signal change across 2-12 seconds (TR 2-6) time 
period poststimulus onset was identified within each region and for each scene valence type (high arousal positive and low arousal negative) and memory content type (scenes with later selective item memory, and scenes with later item and background memory). These signal change values, one per participant per valence/memory content type per region, were used to construct a correlation matrix and a functional model for each age group in each SEM analysis.

The SEM analyses contrasted young and older adults' connectivity that corresponded with later memory performance, either in terms of selectively remembering the emotional item and forgetting its background, or remembering both the emotional item and its background context. Even though these forms of memory differ in selectivity, both are instances of successful memory. As such, the results are not biased by any age differences in the overall levels of encoding success. Also, as age differences in connectivity may vary across valences, connectivity was modeled separately for negative and positive scenes that did not differ by arousal (i.e., negative low arousal items and positive high arousal items). Thus, 4 different SEM analyses were computed, each comparing the connectivity between age groups for: (1) selective item memory for (high arousal) positive scenes, (2) selective item memory for (low arousal) negative scenes, (3) remembering (high arousal) positive item and its background, and (4) and remembering (low arousal) negative item and its background. For each analysis, correlation matrices were created for each condition and age group (e.g., selective memory for low arousal negative items in young adults) using percent signal data extracted for the relevant conditions for each person, revealing the correlations between the regions in the model. Group differences (e.g., between young and older adults) therefore reflect differences across individuals as the activity in 1 region increases.

LISREL 8.7 (Scientific Software International, Inc., Lincolnwood, IL, U.S.A.; Joreskog and Sorbom, 1993) was used to derive estimates of path coefficients from the correlation between neural activity in each region that corresponds with subsequently remembering the item and background from a scene or remembering just the item while forgetting the background for each valence condition. In the null model for each condition, the path coefficients of connections between regions were set to be equal between the two age groups. The null model was then compared with an alternative model where the path coefficients were allowed to vary. The pathways' coefficients can be asymmetric (region A can influence region B differently from the influence of region B upon region A), providing information about the directionality of direct and indirect interregional interactions (Addis and McAndrews, 2006). The fit of the model was assessed by comparison of goodness-of-fit $\chi^{2}$ values, producing a $\chi^{2}$ difference ( $\chi^{2}$ diff $)$ value. A significantly lower $\chi^{2}$ value for the alternative model than the null model indicates that the alternative model is a better fit for the data, indicating the presence of significant age differences in effective connectivity for that specific memory outcome. It is important to note that group differences in model fit, rather that absolute model fit, are critical in this application of SEM. A model of neural activity is necessarily constrained, because only a few influences upon a region can be included, and so the overall model fit may be poor. Even so, inferences about differences between groups are valid regardless of the absolute model fit (Protzner and McIntosh, 2006).

When the alternative model is significantly different from the null model, the connections are tested with iterative fitting of each pathway connection (McIntosh and Gonzalez-Lima, 1994) to determine which connections contribute to the group differences. Connections were set to vary in a stepwise manner, and allowed to remain so only if they improved the overall fit of the model (as determined by a greater $\chi^{2}$ diff). As the order in which connections are allowed to vary can affect which pathways appear to add a significantly better fit to the model, the connections were allowed to vary in 4 different orders (beginning with anterior connections and moving posteriorly, beginning with posterior connections moving 
anteriorly, moving outward from medial to lateral connections, and inward from lateral to medial connections), and the order leading to the best model fit was selected (Addis et al., 2010). The final models for each valence type and memory outcome were stable, with stability indexes $<1$. (The connections identified as significantly contributing to the fit of the model were generally the same regardless of entry order of the pathway into the model, so we depicted the connection order that was most stable and led to the best-fitting model for each condition.) Allowing connections entry to vary from posterior to anterior led to the best fit for the selective positive item memory data, entering connections from anterior to posterior led to the best fit for selective negative item memory data, and the positive item and background remembered data, and entering connections from lateral to medial pathways led to the best fit for the negative item and background remembered data.

\section{Results}

\subsection{Behavioral results}

Behavioral analyses compared the effects of scene valence and age group on the likelihood of successfully remembering the background from a scene, in instances when the item was remembered (Table 2; corrected recognition values are available in Supplementary Table 1). (Because memory for items and backgrounds were tested independently, there is no simple means for subtraction of false alarms from raw recognition values that aligns with the encoding stage neural data corresponding with memory for the item with its background, i.e., [true recognition for items with their backgrounds] - [false alarms to items + false alarms to backgrounds] would be conceptually unrelated to the neuroimaging analyses.)

Analyses were conducted on these data because they most closely aligned with the neuroimaging data for subsequently remembered scenes. We conducted an analysis of variance (ANOVA) on the number of scenes recognized, with factors of memory (item and background remembered, only item remembered and background forgotten), scene emotionality (positive, negative, neutral), and age group (young, older adults). Results showed main effects of emotionality $(F(2,34)=10.19 ; p<0.001)$, group $(F(1,35)=12.60 ; p$ $=0.001)$, and memory $(F(1,35)=4.08 ; p=0.05)$, qualified by interactions between memory and group $(F(1,35)=4.62 ; p<0.05)$ and emotionality and group $(F(2,34)=3.55 ; p<0.05)$. The interaction of memory and group reflects that the age difference in memory (with young adults showing better memory than older adults) were larger when both the item and its background were recognized than when only the item was recognized. The interaction of emotionality and group reflects the fact that young adults showed an enhancement in recognition for positive and negative items compared with neutral items, while older adults showed an enhancement only for the positive items. This is one instantiation of a positivity effect, whereby young adults showed a general enhancement in memory for emotional greater than neutral information, while older adults' memories showed a shift away from negative information relative to the young adults.

Although there were no significant age differences in the total number of scenes for which young and older adults remembered the emotional (positive or negative) item and forgot its background ( $t \mathrm{~s}(35)<1.07 ; p \mathrm{~s}>0.29$ ), young adults remembered more items overall than did older adults $(t \mathrm{~s}(35)>2.53 ; p \mathrm{~s}<0.02)$. Therefore, background memory was analyzed only for the scenes from which the emotional item was remembered; the proportion of these scenes from which only the item (and not the background) was remembered is referred to as the incidence of selective item memory (see right column of Table 2). An ANOVA on the incidence of selective item memory with factors of age (young, older) and emotionality (positive, negative) revealed a main effect of age $(F(1,35)=4.01 ; p=0.05)$ with older adults showing a greater incidence of selective item memory than young adults. There was no main effect of emotion or significant interaction of the factors of emotionality and group $(F(1,35)$ 
$<1)$. In other words, when older adults remembered emotional items, they were significantly more likely to forget the background than were young adults.

An ANOVA on corrected recognition rates of remembering only the background (and forgetting the item) with factors of valence (positive, negative, neutral) and group (young, older) revealed main effects of valence $(F(2,34)=9.48 ; p=0.001)$ and of group $(F(1,35)=$ $7.88 ; p<0.01)$, and trend toward interaction between these factors $(F(2,34)=3.09 ; p=$ $0.06)$. The main effect of group reflects that older adults remembered backgrounds while forgetting the emotional item from the scene more than young adults (older adults $M=5.15$; young adults $M=3.37)$. The main effect of valence shows that remembering only the background occurs most often for neutral scenes $(M=5.83)$ than negative $(M=3.88)$ or positive scenes $(M=3.07)$. However the trending interaction shows that there were differences between age groups only for the negative scenes $(t(35)=3.23 ; p=0.004$; positive and neutral scenes $t \mathrm{~s}(35)<1.34 ; p \mathrm{~s}>0.19)$

\subsection{Connectivity model evaluation}

Omnibus tests of the difference in path coefficients between the null and alternate models revealed significant age differences in the connectivity for each of the 4 models of the memory outcomes for positive and negative scenes (selective positive item memory [ $\chi^{2}$ diff $=$ 34.29; $p<0.0001$ ]; selective negative item memory [ $\chi^{2}$ diff $\left.40.55 ; p<0.0001\right]$; positive item and background remembered [ $\chi^{2}$ diff $\left.=35.09 ; p=1.08 \mathrm{e}-05\right]$; negative item and background remembered [ $\chi^{2}$ diff $\left.=30.20, p=3.6 \mathrm{e}-05\right]$; beta weights are available in Supplementary Table 2). Fig. 3 shows the neural connectivity present during encoding that corresponds with later remembering both the item and background components from the scene (positive scenes shown in Fig. 3A and B; negative scenes shown in Fig. 3C and D). Fig. 4 shows the neural connectivity present during encoding that corresponds with a later selective emotional item memory (positive scenes shown in Fig. 4A and B; negative scenes shown in Fig. 4C and D).

3.2.1. Activation patterns for scene memory-Specific observations from these models are detailed below. While we focus on connectivity results below, the results of analyses comparing activation patterns are available in Supplementary Table 3. An ANOVA of the encoding-related activation data with factors of memory [item only subsequently remembered, item and background subsequently remembered], emotion [high arousal positive, low arousal negative], and age [young adults, older adults] revealed a main effect of age, but no main effects of memory or emotion $[p<0.001 ; 5$ voxel extent; see Supplementary Table 3].) Both young and older adults had activation distributed throughout the brain. Young adults showed greater activation than older adults in portions of occipital and prefrontal cortices, while older adults showed greater activation in parietal regions and other areas of occipital cortex. There was a significant 3-way interaction within left inferior frontal gyrus (BA 47; $-40,21,-4)$ and midbrain subthalamic nucleus $(6,-10,-5 ; p<0.001$; 5 voxel extent). Further examination of this interaction revealed that only older adults showed activity corresponding with the interaction of memory and emotion, within the left inferior frontal gyrus (BA 47; -40, 23, -1) and posterior cingulate (BA 30; 6, -48, 17) (young adults did not show any clusters surpassing threshold for this interaction). Notably, there were no regions that showed 2-way interaction between memory and age, suggesting that the age differences may have been related to general scene processing rather than to memory-specific processes. More generally, the activation profile emphasizes that older adults' greater connectivity within right-sided frontal regions, is not merely reflective of an accompanying age-specific increase in activation. 


\subsection{Age differences in connectivity corresponding with successful memory for item and background}

Consistent with PASA, older adults show significantly stronger connectivity for pathways involving frontal regions. For instance, even though young and older adult groups both show strong positive connectivity between orbitofrontal and dorsolateral prefrontal regions corresponding with remembering either positive or negative items with their backgrounds, this connectivity is significantly stronger in older adults than young adults when encoding positive items with backgrounds (Fig. 3B and D). Older adults also show strong positive right orbitofrontal-left amygdala connectivity relative to young adults for both scene valences (Fig. 3A and C). The simple correlations between each individual's levels of activity in the left amygdala and the right OFC further illustrate the age-related changes in the correspondence between these two regions. There is a significant age difference in the strength of the correlations for positive scenes $(z=3.15 ; p=0.002)$ and for negative scenes ( $z=2.09 ; p=0.04$ ), with the direction of the correlations reversing in the two age groups. There is a positive relationship between left amygdala and right orbitofrontal activity in older adults (positive scenes $r=0.52 ; p<0.05$; negative scenes $r=0.52 ; p<0.05$ ), and a negative relationship in young adults, although that relationship only reaches significance for the positive scenes (positive scenes $r=-0.52 ; p<0.05$; negative scenes $r=-0.19 ; p=$ 0.47; Fig. 5, upper panel).

In contrast to older adults' enhanced connectivity between the left amygdala and right OFC, older adults show some instances of negative connectivity as compared with young adults, particularly within more posterior regions; the amygdala-fusiform pathways that significantly differed between age groups had negative beta-weights in older adults and positive beta-weights in young adults (see Supplementary Table 2). This is true for both positive and negative scenes, evident in the pathway from right fusiform to left amygdala during encoding of positive scenes, and in the pathway from the left amygdala to left fusiform for negative scenes (comparison Fig. 3A and B, and comparison Fig. 3C and D). However, not all of the posterior connections that differ significantly between young and older adults among posterior regions are negative in older adults; older adults have significantly stronger positive connectivity than young adults between the left fusiform and left superior parietal lobule for successful encoding of scenes of both valences, but particularly for negative scenes.

\subsection{Age differences in connectivity corresponding with selective emotional item memory}

When examining the neural connectivity corresponding with selective memory for the emotional items (and not their backgrounds), one notable difference was that older adults no longer showed the same shift toward strengthened amygdala-orbitofrontal connectivity. In contrast to what had been revealed in cases of encoding the item and the background, the correlation strengths in the two groups did not significantly differ in cases of selective item memory - and the direction of the correlations trended in the opposite direction-being positive in the young adults and negative in the older adults (for positive scenes: $z=1.48 ; p$ $=0.14$; young adults $r=0.25 ; p=0.33$; older adults $r=-0.28 ; p=0.27$; for negative scenes: $Z=1.54 ; p=0.12$; young adults $r=0.45 ; p=0.07$; older adults $r=-0.08 ; p=0.76$ ).

Visual inspection of the connectivity models revealed distinct patterns of age differences for positive and negative scenes. For negative scenes, young adults recruit the left amygdala into the neural network corresponding with selective negative item memory to a greater extent than older adults. Young adults have particularly strong connections from the left amygdala to right OFC, left parahippocampus, and right fusiform (Fig. 4C). In contrast, the left amygdala has no strong connectivity with these areas in older adults within the network corresponding with selective negative item memory, and in fact the relation is often in a 
negative direction (e.g., increased activity in the left amygdala is associated with decreased activity in the right OFC or right fusiform gyrus; Fig. 4D).

For selective item memory in positive scenes, young and older adults share moderate connectivity from the left amygdala to the right OFC (Fig. 4A and B). However, there is some evidence of enhanced frontal connectivity in older adults; the older adults have strong connectivity between orbitofrontal and dorsolateral prefrontal regions, whereas these frontal regions are not strongly connected in young adults. There also is some evidence of weakened posterior connectivity in the older adults, with the older adults showing no strong positive connections between the left or right fusiform gyrus and the left amygdala.

\section{Discussion}

This study examined age differences in the neural connectivity engaged during the encoding of scenes including an emotional item. The results revealed that relative to young adults, older adults showed enhanced frontal connectivity when they broadened their attentional focus and successfully encoded both the item and background components of the scene. However, this age-related anterior shift in connectivity was not evident during selective memory encoding of the emotional item in a scene. The results suggest that greater frontal connectivity may provide a compensatory mechanism for older adults, enabling them to successfully encode items and backgrounds of emotional scenes.

\subsection{Age differences in connectivity underlying successful encoding of both item and background}

Building upon the literature examining memory for complete emotional images, results showed that in older adults strong positive connections among frontal regions and a general shift to more anterior processing correspond with successful memory for emotional items and their studied backgrounds, regardless of whether the scenes were of positive or negative valence. The enhanced frontal connectivity in older adults is consistent with PASA (Davis et al., 2008; Dennis et al., 2008), which likely reflects changes in older adults' encoding strategies or compensatory prefrontal connectivity to offset declining functionality in more posterior visual processing regions. Interestingly, the activation data do not reveal the shift described by PASA. The ANOVA of the neural activation related to memory for the emotional scenes showed a broad distribution of regions selectively active for either young or older adults regardless of scene type or emotionality, however there were no interactions among the factors of age, memory, and emotion, and no evidence that older adults disproportionately recruited the prefrontal cortex.

We are not the first to find a disconnect between activation and neural connectivity; Dennis et al. (2008) also reported that although there was enhanced frontal connectivity in older adults, prefrontal activation was reduced in older adults compared with young adults (see also Della-Maggiore et al., 2000 for a similar age-related disconnect between results of hippocampal regional cerebral blood flow $[\mathrm{rCBF}]$ and networking). As pointed out by Grady (2005), behavioral, activation, and connectivity analyses do not always paint the same picture; some types of analyses may detect effects that are not apparent with other methods. When there are similar patterns of activation supporting memory performance in young and older adults, differences in connectivity between regions may explain age-related differences in memory performance.

In terms of emotional scene processing, the current findings suggest that enhanced prefrontal connectivity in older adults enables them to encode the background as well as the item within the emotional scene. More generally, this study provides further evidence that an agerelated shift in connectivity occurs for encoding of emotional as well as neutral information 
(also see Murty et al., 2009; St. Jacques et al., 2010). In the current study, this frontal shift in older adults may underpin controlled efforts to broaden attention toward the background portions of the scene when faced with an emotional item, resulting in successful encoding of both the emotional item and the neutral background composing the scene. This hypothesis is consistent with the cognitive control hypothesis of aging, whereby older adults' attention and memory is self-directed by emotion-regulation goals when confronted with emotional information or experiences (reviewed by Nashiro et al., 2012). As described by St. Jacques et al. (2009a), age-related increases in the engagement of frontal brain regions may reflect either neural compensation or emotion regulation, and these explanations are not at odds; in fact emotion regulation could be a method of compensation. In young adults, attention to the background in the presence of an emotional item may not require such strategic attentional processes. Without eye-tracking data, inferences about whether this processing reflects additional visual search efforts remain speculative, but provide an important direction for future research.

This is the first study to show that older adults demonstrate enhanced prefrontal connectivity compared with young adults for both negative and positive scenes. Some studies have found that older adults' prefrontal connectivity is stronger when encoding positive than negative information (Addis et al., 2010), but others have observed this connectivity pattern when examining connectivity solely for negative stimuli (St. Jacques et al., 2009b, 2010; Murty et al., 2009). Taken together, the results of this study and the current literature suggest that older adults' enhanced pre-frontal connectivity may reflect a general age-related shift in the neural connectivity supporting successful episodic memory for neutral (Dennis et al., 2008; for a review see Grady, 2008) and emotional images, and may not be valence-specific.

It is worthwhile to compare the results of this study to those reported by Addis et al. (2010), the only other study examining age differences in the effective connectivity underlying successful memory for positive as well as negative images. Although positive and negative scenes were not directly compared, it is interesting to note that older adults show moderate to strong connectivity among orbitofrontal and dorsolateral prefrontal regions while successfully encoding positive items, regardless of whether the accompanying background in the scene was subsequently remembered or forgotten. This finding is generally consistent with Addis et al. (2010) who found that older adults showed intensified prefrontal connectivity only for positive (and not for negative) information. However, Addis et al. (2010) found that age differences were restricted to the connectivity corresponding with memory for positive scenes, whereas the present study showed significant age differences underlying negative as well as positive scene encoding. The stimulus and task parameters differed in these studies, as did the regions and pathways included in the models, so it is not surprising that the connectivity differed in several respects as well. The comparisons of these two studies suggest that enhanced connectivity between prefrontal regions generalizes across a variety of tasks involving the encoding of positive information, whereas age differences in the encoding of negative information may be most apparent in pathways that are task-specific.

Because the frontal lobe encompasses a large and highly interconnected area of the human cortex, and has been shown to be necessary for a number of higher-order processes (reviewed by Duncan and Owen, 2000; Smith and Jonides, 1999; Stuss, 2011), it is prudent to consider specific functions of the subregions that are specifically relevant to emotional memory. Regions of the prefrontal cortex, including dIPFC and ventrolateral prefrontal cortex (vlPFC), are routinely identified in studies investigating the activation and connectivity underlying older adults' memory for neutral and emotional stimuli (Dennis et al., 2008; St. Jacques et al., 2009b). Yet it is the OFC that is often implicated specifically in the encoding of emotional (and not neutral) information, and several studies have also 
implicated the OFC in the encoding of emotionally salient visual stimuli (Kensinger and Schacter, 2006, 2008; Kensinger et al., 2007a), perhaps because the OFC plays an integral role for evaluation of emotionally salient stimuli within a visual context (Kringelbach and Rolls, 2004; Rolls, 2004). Consistent with a role for the OFC in visual identification of emotion, a study of patients with neurodegenerative diseases showed that gray matter volume in a very similar region of the right lateral OFC as that identified in the present study correlated with the ability to accurately evaluate emotional expression in a dynamic stimulus (Goodkind et al., 2012). The OFC also is also linked to the regulation of emotion and to the modulation of autonomic responses (Cavada et al., 2000; reviewed by Hooker and Knight, 2006). The enhanced connectivity between the left amygdala and OFC observed in older adults in the present study, therefore, may reflect the emotional evaluation and regulation processes proposed within the cognitive control hypothesis, which states older adults place a greater focus upon regulating their emotional reactions than do young adults (reviewed by Nashiro et al., 2012; Ochsner and Gross, 2005). The fact that in the present study older adults demonstrated stronger and more positive connectivity between OFC and dIPFC and also between OFC and the amygdala may be evidence of greater reliance upon OFC evaluative and regulatory processes for emotional scene memory by older adults than young adults.

\subsection{Age differences in connectivity associated with selective memory}

The age-related intensification of positive prefrontal-amygdala connectivity that occurred during holistic scene encoding of item and background did not extend to instances in which only the emotional item was successfully encoded. In fact, the connectivity corresponding with older adults' selective memory showed that the amygdala had largely negative connections with the other nodes of the network, including prefrontal regions. Thus, even when the prefrontal-amygdala connections are negative, older adults can still encode the emotionally relevant information but at the expense of encoding the context. Taken together, these results suggest that positive prefrontal-amygdala connections may provide a pathway for older adults to concurrently encode both item and background components of the scene.

These results can be considered in the context of the behavioral results, which reveal that when the item is remembered, older adults have a greater propensity to forget the backgrounds of emotional scenes than do young adults. This finding is consistent with prior research indicating that older adults can have more difficulty than young adults at encoding the backgrounds accompanying an emotional item (Kensinger et al., 2007d). Older adults are more susceptible to variations in attention, and emotional information in particular may draw their attention, leading to reduced ability to integrate both the emotional item and the background into memory. It is conceivable that aging results in negative amygdala connectivity and that this change makes older adults more susceptible to forgetting the background of a scene when they remember the emotional item. When older adults do successfully encode the item and background portions of the scenes, it may be limited to trials when they employed more controlled efforts to deploy attention toward the background portions of the scene, however this remains to be tested in future studies.

Previous literature has shown that the amygdala may function as a salience or relevance detector, activating in response to novel, significant, or arousing information of positive and negative valences (Hamann et al., 1999; Sander et al., 2003). In the present study, older adults had negative amygdala connectivity during encoding of selectively remembered negative items within pathways where young adults instead had strongly positive connectivity; this included left amygdala connections with the left parahippocampus, right $\mathrm{OFC}$, and right fusiform (the left fusiform pathway was also very weak, although not significantly different from young adults). One possibility is that differences in connectivity may reflect that older adults find less relevance in negative items than do young adults. This 
study did not draw direct comparison between older adults' connectivity for negative versus positive scenes, however, the possibility of diminished relevance for negative information in older adults is supported by previous literature showing reduced amygdala connectivity underlying negative information encoding in older adults with higher compared with lower life satisfaction (Waldinger et al., 2011). In the present study, age differences in the connectivity underlying selective negative item memory may reflect lesser relevance of this information to older adults compared with young adults, even when it is later remembered.

\subsection{Future directions}

These data provide a starting point toward understanding how the connectivity underlying selective and holistic memory differs between young and older adults for positive and negative information. Although the connectivity model used in the present study included regions involved in emotional processing and memory, a broader network including valence-specific regions and a distribution of regions across the prefrontal cortex may more completely capture the neural response corresponding with age differences in the encoding of emotional scenes.

In future studies, it may be worthwhile to contrast connectivity between groups of stimuli categorized using the participant's own ratings of stimulus valence and arousal. There may be some differences in connectivity that are apparent for those stimuli the individual finds most arousing, which are no longer evident when grouped with less arousing stimuli. Moreover, although the arousal ratings for the sets of positive and negative stimuli did not significantly differ, arousal may not directly proxy their degree of saliency. Positive stimuli in particular may hold a saliency in memory that is not captured by arousal ratings alone. Positive emotion related to satisfying desire contains a motivational component that promotes attention narrowing to shut out information irrelevant to goal attainment, while positive emotion resulting from a sense of pleasure after attaining a reward more often broadens attentional focus allowing more flexible connections between pieces of information (Levine and Edelstein, 2009). It may be fruitful to consider the nuances of qualitative meaning in what high and low arousal signal differently for positive and negative stimuli. It also may be beneficial for future studies to attempt to tease apart emotional content from item content by contrasting the memory effects of emotionally arousing contexts containing neutral items to the memory effect of neutral contexts containing emotionally arousing items in order to more fully understand the independent contributions of the factors of emotion and scene component.

\subsection{Conclusions}

The results described here help to explain how selective and holistic memory for emotional visual information arises in young and older adults. We found significant age differences in the encoding-related neural connectivity of subsequently remembered components of positive and negative scenes. These age differences extend to successful encoding of both item and background and also selective memory for emotional items. In instances where the background is remembered as well as the item, older adults exhibit stronger positive connectivity among frontal regions and from pre-frontal to medial temporal regions. This finding contrasted with young adults' more posterior pattern of connectivity between fusiform and medial temporal regions. Together, this pattern is consistent with a compensatory shift to pre-frontal-based emotion processing with aging; this shift may enable older adults to encode more aspects of complex scenes. The prefrontal-amygdala connectivity underlying selective item memory also differed between young and old, suggesting that this phenomenon may reflect different neural processes at encoding depending on age. By highlighting the pathways of processing that lead to effective information encoding, investigations of neural connectivity changes in aging such as the one 
reported here may help to guide future research regarding the techniques that are most effective for improving memory at each age period, such as study techniques for young adults or cognitive enhancement and rehabilitation in older adults.

\section{Supplementary Material}

Refer to Web version on PubMed Central for supplementary material.

\section{Acknowledgments}

This research was supported by grants from the National Institute of Mental Health (MH080833) (to EAK), the American Psychological Association (to JDW) and American Psychological Foundation/COGDOP (to JDW). Portions of this report were included in the doctoral dissertation of J.D.W. The authors thank Ehri Ryu, Hiram Brownell, and Anne Krendl for helpful discussions, and Keely Muscatell and Kristin Hricko for assistance with data collection.

\section{References}

Addis DR, Leclerc CM, Muscatell KA, Kensinger EA. There are age-related changes in neural connectivity during the encoding of positive, but not negative, information. Cortex. 2010; 46:425433. [PubMed: 19555933]

Addis DR, McAndrews MP. Prefrontal and hippocampal contributions to the generation and binding of semantic associations during successful encoding. NeuroImage. 2006; 33:1194-1206. [PubMed: 17023179]

Addis DR, Moscovitch M, McAndrews MP. Consequences of hippocampal damage across the autobiographical memory network in left temporal lobe epilepsy. Brain. 2007; 130:2237-2342.

Amaral DG, Behniea H, Kelly JL. Topographic organization of projections from the amygdala to the visual cortex in the macaque monkey. Neuroscience. 2003; 118:1099-1120. [PubMed: 12732254]

Brett, M.; Anton, J.; Valabregue, R.; Poline, J. Region of interest analysis using an SPM toolbox [Abstract]. CD-ROM in NeuroImage; Presented at the 8th International Conference on Functional Mapping of the Human Brain; Sendai, Japan. 2002. available on

Burke A, Heuer F, Reisberg D. Remembering emotional events. Mem Cogn. 1992; 20:277-290.

Cavada C, Compañy T, Tejedor J, Cruz-Rizzolo RJ, Reinoso-Suárez F. The anatomical connections of the macaque monkey orbitofrontal cortex. A review Cereb Cortex. 2000; 10:220-242.

Chee MW, Goh JO, Venkatraman V, Tan JC, Gutchess A, Sutton B, Hebrank A, Leshikar E, Park D. Age-related changes in object processing and contextual binding revealed using fMR adaptation. $J$ Cogn Neurosci. 2006; 18:495-507. [PubMed: 16768356]

Davis SW, Dennis NA, Daselaar SM, Fleck MS, Cabeza R. Que PASA? The posterior-anterior shift in aging. Cereb Cortex. 2008; 18:1201-1209. [PubMed: 17925295]

Della-Maggiore V, Sekuler AB, Grady CL, Bennett PJ, Sekuler R, McIntosh AR. Corticolimbic Interactions Associated with Performance on a Short-Term Memory Task Are Modified by Age. J Neurosci. 2000; 20:8410-8416. [PubMed: 11069948]

Dennis NA, Hayes SM, Prince SE, Madden DJ, Huettel SA, Cabeza R. Effects of aging on the neural correlates of successful item and source memory encoding. J Exp Psychol Learn Mem Cogn. 2008; 34:791-808. [PubMed: 18605869]

Duncan J, Owen AM. Common regions of the human frontal lobe recruited by diverse cognitive demands. Trends Neurosci. 2000; 23:475-483. [PubMed: 11006464]

Gazzaley A, D’Esposito M. Top-Down Modulation and Normal Aging. Ann N Y Acad Sci. 2007; 1097:67-83. [PubMed: 17413013]

Goodkind MS, Sollberger M, Gyurak A, Rosen HJ, Rankin KP, Miller B, Levenson R. Tracking emotional valence: the role of the orbitofrontal cortex. Hum Brain Mapp. 2012; 33:753-762. [PubMed: 21425397]

Grady, CL. Cognitive Neuroscience of Aging; Linking Cognitive and Cerebral Aging. Oxford University Press; New York: 2005. Functional connectivity during memory tasks in healthy aging and dementia; p. 286-308. 
Grady CL. Cognitive neuroscience of aging. Ann N Y Acad Sci. 2008; 1124:127-144. [PubMed: 18400928]

Gutchess AH, Hebrank A, Sutton BP, Leshikar E, Chee MW, Tan JC, Goh JO, Park DC. Contextual interference in recognition memory with age. NeuroImage. 2007; 35:1338-1347. [PubMed: 17355910]

Gutchess AH, Welsh RC, Hedden T, Bangert A, Minear M, Liu LL, Park DC. Aging and the neural correlates of successful picture encoding: frontal activations compensate for decreased medialtemporal activity. J Cogn Neurosci. 2005; 17:84-96. [PubMed: 15701241]

Hamann S. Cognitive and neural mechanisms of emotional memory. Trends Cogn Sci. 2001; 5:394400. [PubMed: 11520704]

Hamann SB, Ely TD, Grafton ST, Kilts CD. Amygdala activity related to enhanced memory for pleasant and aversive stimuli. Nat Neurosci. 1999; 2:289-293. [PubMed: 10195224]

Hasher, L.; Zacks, RT. Working memory, comprehension, and aging: a review and a new view. In: Bower, G., editor. The Psychology of Learning and Motivation. Vol. 22. Academic Press; New York: 1988. p. 193-225.

Hooker, CI.; Knight, RT. The role of lateral orbitofrontal cortex in the inhibitory control of emotion. In: Zald, DH.; Rauch, SL., editors. The Orbitofrontal Cortex. Oxford University Press; New York: 2006. p. 307-324.

Joreskog, K.; Sorbom, D. Scientific, Software International. Chicago: 1993. LISREL 8: Users' Reference Guide.

Kensinger EA, Garoff-Eaton RJ, Schacter DL. Memory for specific visual details can be enhanced by negative arousing content. J Mem Lang. 2006; 54:99-112.

Kensinger EA, Garoff-Eaton RJ, Schacter DL. How negative emotion enhances the visual specificity of a memory. J Cogn Neurosci. 2007a; 19:1872-1887. [PubMed: 17958489]

Kensinger EA, Garoff-Eaton RJ, Schacter DL. Effects of emotion on memory specificity in young and older adults. J Gerontol B. 2007b; 62:208-215.

Kensinger EA, Garoff-Eaton RJ, Schacter DL. Effects of emotion on memory specificity: Memory trade-offs elicited by negative visually arousing stimuli. J Mem Lang. 2007c; 56:575-591.

Kensinger EA, Gutchess AH, Schacter DL. Effects of aging and encoding instructions on emotioninduced memory trade-offs. Psychol Aging. 2007d; 22:781-795. [PubMed: 18179297]

Kensinger EA, Piguet O, Krendl AC, Corkin S. Memory for contextual details: effects of emotion and aging. Psychol Aging. 2005; 20:241-250. [PubMed: 16029088]

Kensinger EA, Schacter DL. Amygdala activity is associated with the successful encoding of item, but not source, information for positive and negative stimuli. J Neurosci. 2006; 26:2564-2570. [PubMed: 16510734]

Kensinger EA, Schacter DL. Remembering the specific visual details of presented objects: neuroimaging evidence for effects of emotion. Neuropsychologia. 2007; 45:2951-2962. [PubMed: 17631361]

Kensinger EA, Schacter DL. Neural processes supporting young and older adults' emotional memories. J Cogn Neurosci. 2008; 20:1161-1173. [PubMed: 18284340]

Kringelbach ML, Rolls ET. The functional neuroanatomy of the human orbitofrontal cortex: evidence from neuroimaging and neuropsychology. Prog Neurobiol. 2004; 72:341-372. [PubMed: 15157726]

LaBar KS, Cabeza R. Cognitive neuroscience of emotional memory. Nat Rev Neurosci. 2006; 7:5464. [PubMed: 16371950]

Lamar M, Resnick SM. Aging and prefrontal functions: dissociating orbitofrontal and dorsolateral abilities. Neurobiol Aging. 2004; 25:553-558. [PubMed: 15013577]

Levine LJ, Edelstein RS. Emotion and memory narrowing: a review and goal-relevance approach. Cogn Emotion. 2009; 23:833-875.

Libkuman TM, Stabler CL, Otani H. Arousal, valence, and memory for detail. Memory. 2004; 12:237247. [PubMed: 15250188]

Mather M, Sutherland MR. Arousal-biased competition in perception and memory. Perspect Psychol Sci. 2011; 6:114-133. [PubMed: 21660127] 
McIntosh AR. Mapping cognition to the brain through neural interactions. Memory. 1999; 7:523-548. [PubMed: 10659085]

McIntosh AR, Gonzalez-Lima F. Structural equation modeling and its application to network analysis in functional brain imaging. Hum Brain Mapp. 1994; 2:2-22.

Moriguchi Y, Negreira A, Weierich M, Dautoff R, Dickerson BC, Wright CI, Barrett LF. Differential hemodynamic response in affective circuitry with aging: an fmri study of novelty, valence, and arousal. J Cogn Neurosci. 2011; 23:1027-1041. [PubMed: 20521849]

Murty VP, Sambataro F, Das S, Tan HY, Callicott JH, Goldberg TE, Meyer-Lindenberg A, Weinberger DR, Mattay VS. Age-related alterations in simple declarative memory and the effect of negative stimulus valence. J Cogn Neurosci. 2009; 21:1920-1933. [PubMed: 18823239]

Nashiro K, Sakaki M, Mather M. Age differences in brain activity during emotion processing: reflections of age-related decline or increased emotion regulation? Gerontology. 2012; 58:156163. [PubMed: 21691052]

Ochsner KN, Gross JJ. The cognitive control of emotion. Trends Cogn Sci. 2005; 9:242-249. [PubMed: 15866151]

Patterson DW, Schmidt LA. Neuroanatomy of the human affective system. Brain Cogn. 2003; 52:2426. [PubMed: 12812801]

Protzner AB, McIntosh AR. Testing effective connectivity changes with structural equation modeling: what does a bad model tell us? Hum Brain Mapp. 2006; 27:935-947. [PubMed: 16929548]

Reisberg, D.; Heuer, F. Memory for emotional events. In: Reisberg, D.; Hertel, P., editors. Memory and Emotion. University Press; Oxford: 2004. p. 3-41.

Rolls ET. The functions of the orbitofrontal cortex. Brain Cogn. 2004; 55:11-29. [PubMed: 15134840]

Salat DH, Kaye JA, Janowsky JS. Greater orbital prefrontal volume selectively predicts worse working memory performance in older adults. Cereb Cortex. 2002; 12:494-505. [PubMed: 11950767]

Sander D, Grafman J, Zalla T. The human amygdala: an evolved system for relevance detection. Rev Neurosci. 2003; 14:303-316. [PubMed: 14640318]

Smith EE, Jonides J. Storage and executive processes in the frontal lobes. Science. 1999; 283:16571661. [PubMed: 10073923]

Stein JL, Wiedholz LM, Bassett DS, Weinberger DR, Zink CF, Mattay VS, Meyer-Lindenberg A. A validated network of effective amygdala connectivity. NeuroImage. 2007; 36:736-745. [PubMed: 17475514]

St Jacques PL, Bessette-Symons B, Cabeza R. Functional neuroimaging studies of aging and emotion: fronto-amygdalar differences during emotional perception and episodic memory. $\mathrm{J}$ Int Neuropsychol Soc. 2009a; 15:819-825. [PubMed: 19703320]

St Jacques PL, Dolcos F, Cabeza R. Effects of aging on functional connectivity of the amygdala for subsequent memory of negative pictures: a network analysis of functional magnetic resonance imaging data. Psychol Sci. 2009b; 20:74-84. [PubMed: 19152542]

St Jacques P, Dolcos F, Cabeza R. Effects of aging on functional connectivity of the amygdala during negative evaluation: A network analysis of fMRI data. Neurobiol Aging. 2010; 31:315-327. [PubMed: 18455837]

Stuss DT. Functions of the frontal lobes: relation to executive functions. J Int Neuropsychol Soc. 2011; 17:759-765. [PubMed: 21729406]

Swanson LW, Petrovich GD. What is the amygdala? Trends Neurosci. 1998; 21:323-331. [PubMed: 9720596]

Talairach, J.; Tournoux, P. Co-Planar Stereotaxic Atlas of the Human Brain. Thieme Medical; New York: 1988.

Waldinger RJ, Kensinger EA, Schulz MS. Neural activity, neural connectivity, and the processing of emotionally valenced information in older adults: links with life satisfaction. Cogn Affect Behav Neurosci. 2011; 11:426-436. [PubMed: 21590504]

Waring JD, Kensinger EA. Effects of emotional valence and arousal upon memory trade-offs with aging. Psychol Aging. 2009; 24:412-422. [PubMed: 19485658]

Waring JD, Kensinger EA. How emotion leads to selective memory: Neuroimaging evidence. Neuropsychologia. 2011; 49:1831-1842. [PubMed: 21414333] 
A.
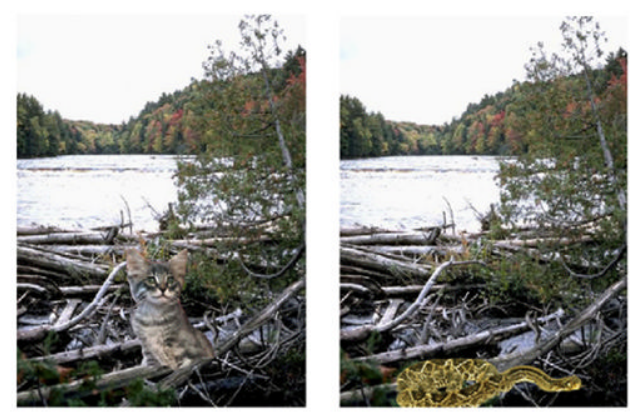

B.

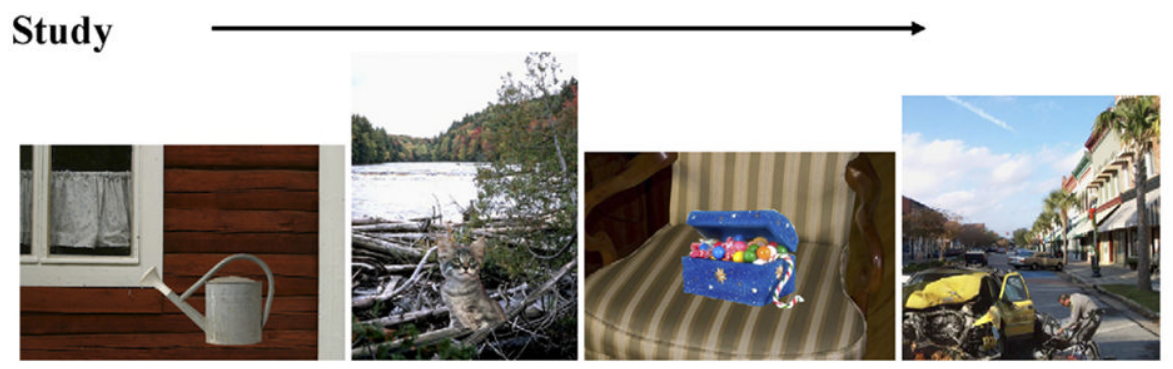

C.

Test

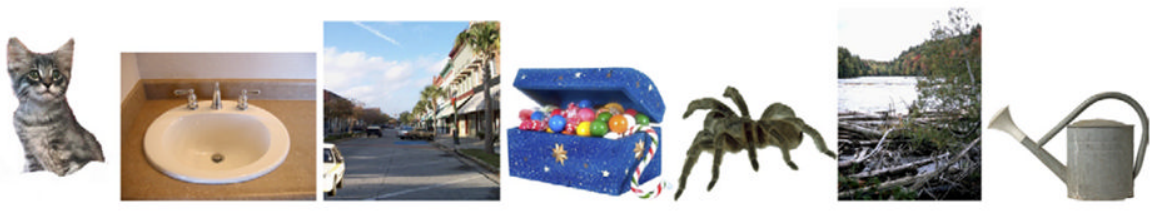

Fig. 1.

Stimulus exemplars. Example of positive (kitten) or negative (snake) items placed in the context of a neutral background (river; A). Examples of studied scenes (B), and items and backgrounds presented separately at test (C). 


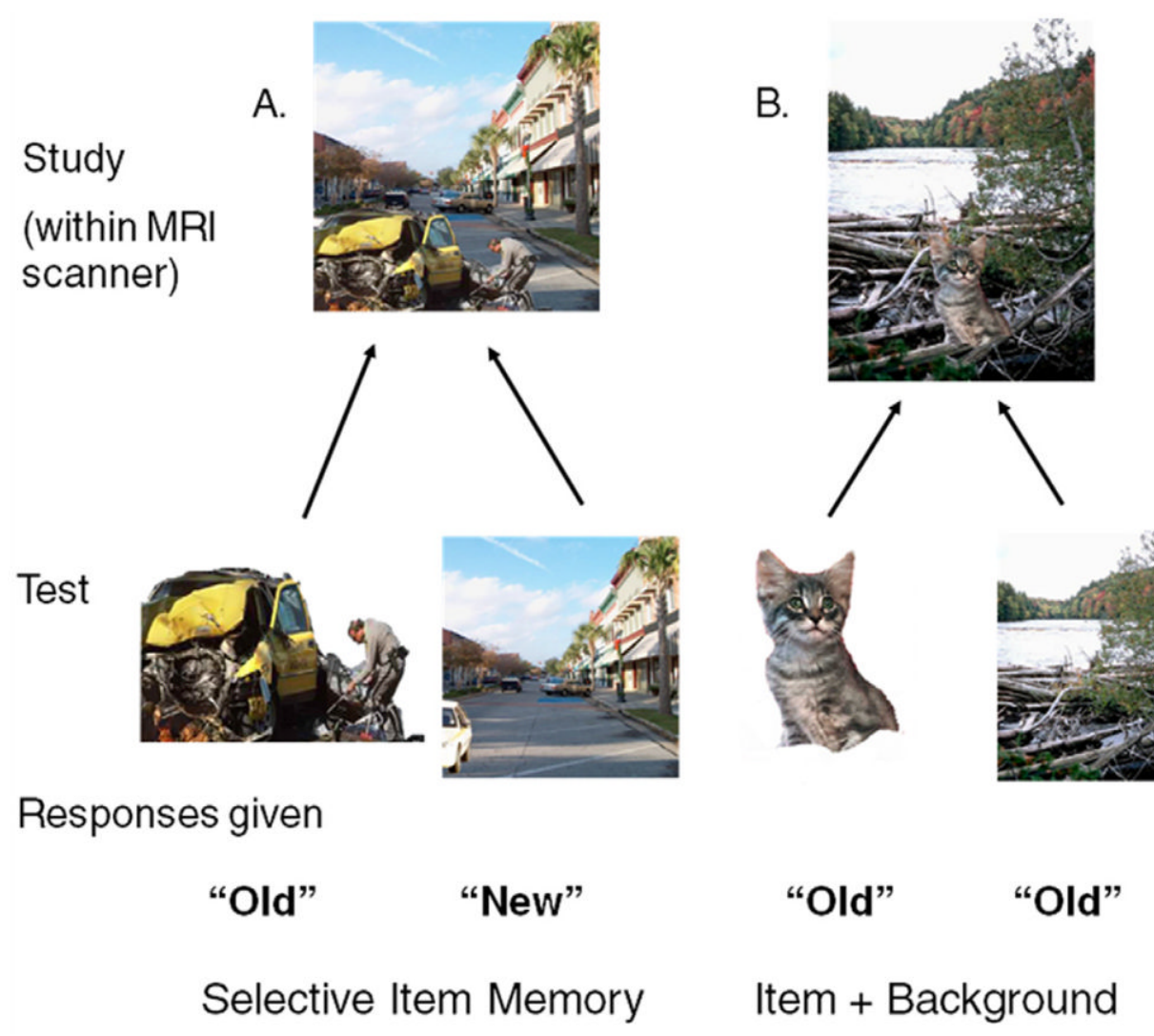

Fig. 2.

Subsequent Memory Analyses of Participant Responses. (A) Subsequent selective memory for the item (car crash) and forgetting the background (avenue). (B) Subsequent memory for the item (kitten) and the background (river). 


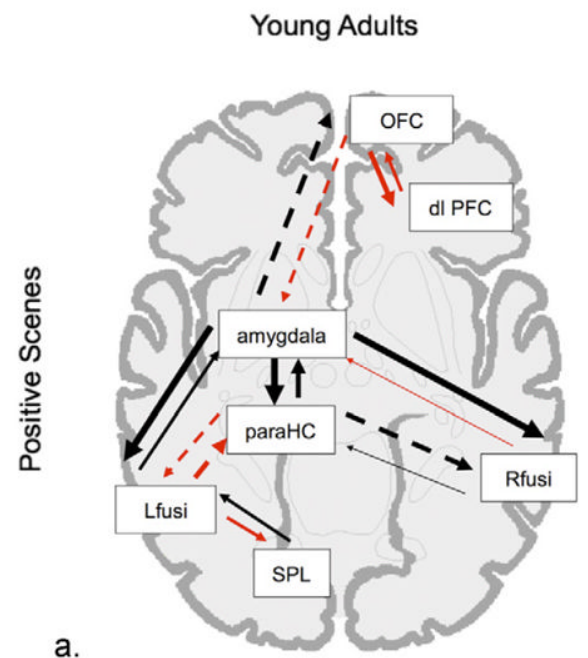

a.

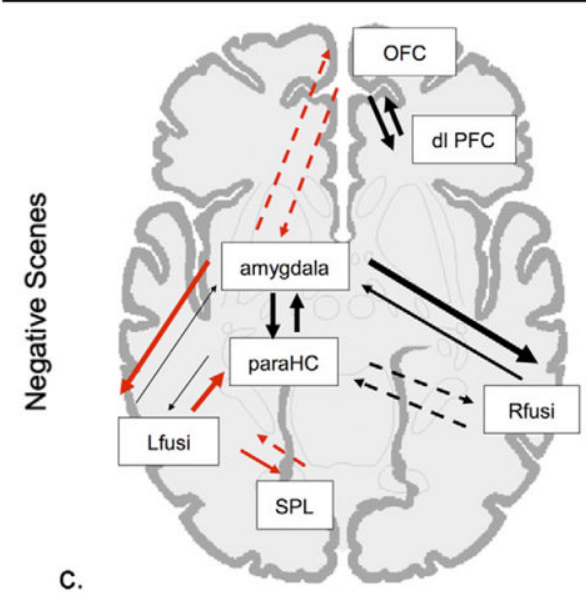

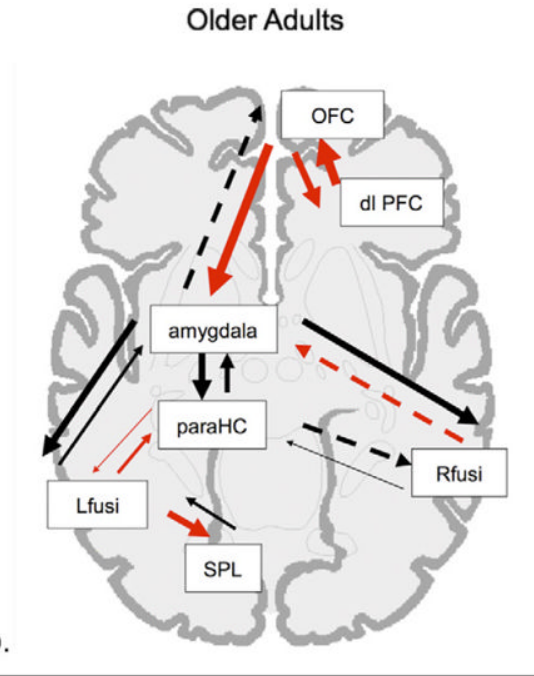

b.

d.

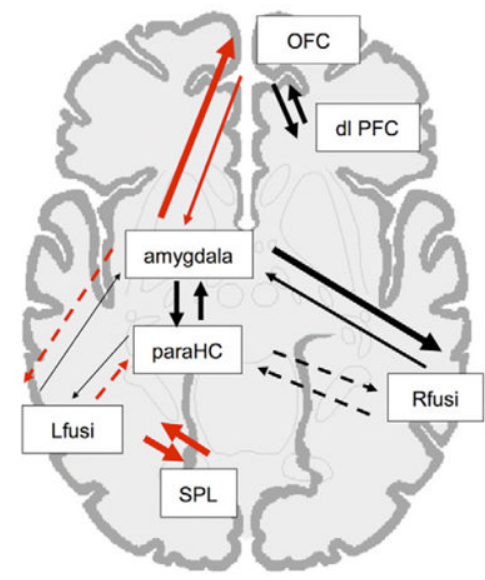

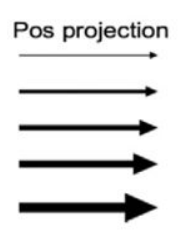

Beta Weights

0.0-0.1

0.1-0.2

0.2-0.4

0.4-0.6

0.6-1.0
Neg projection

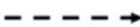
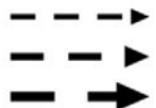

Fig. 3.

Model of encoding-related connectivity for remembering item and background. Visual depiction of the beta weights of path coefficients of functional networks related to successful memory for emotional items and their backgrounds. As shown in legend, thicker arrows signify stronger connections between regions, solid arrows signify positive projections, and dashed arrows signify negative projections. Red arrows denote paths where coefficients for young and older adults significantly differ from each other. (a) Young adults positive scenes, (b) older adults positive scenes, (c) young adults negative scenes, (d) older adults negative scenes. Abbreviations: dlPFC, dorsolateral prefrontal cortex; Lfusi, left fusiform; OFC, orbitofrontal cortex; paraHC, parahippocampus; Rfusi, right fusiform; SPL, superior parietal lobule. 


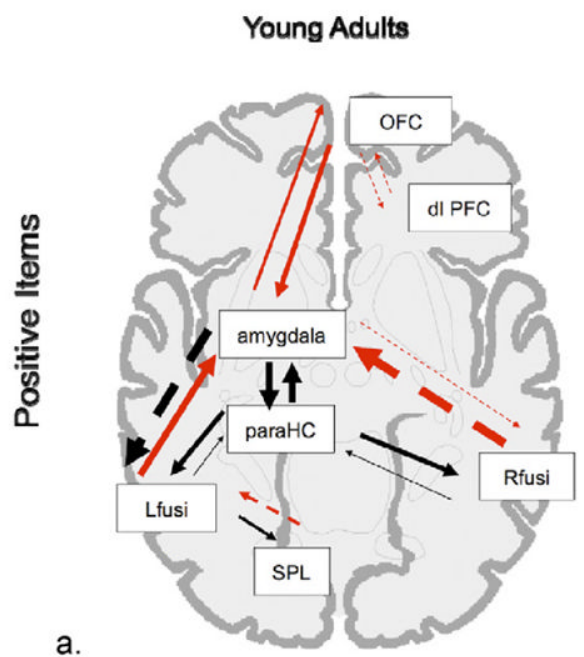

b.
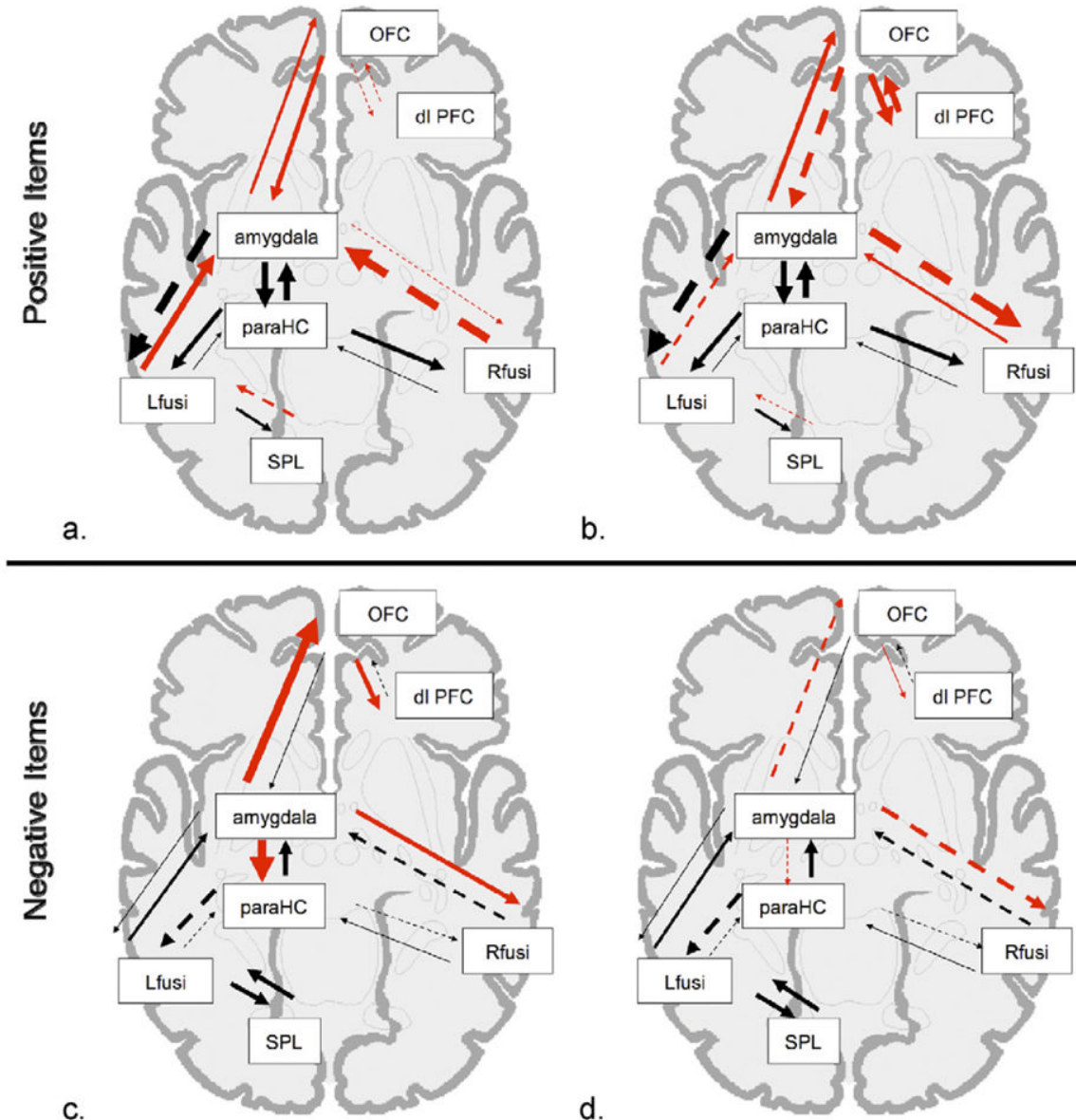

d.
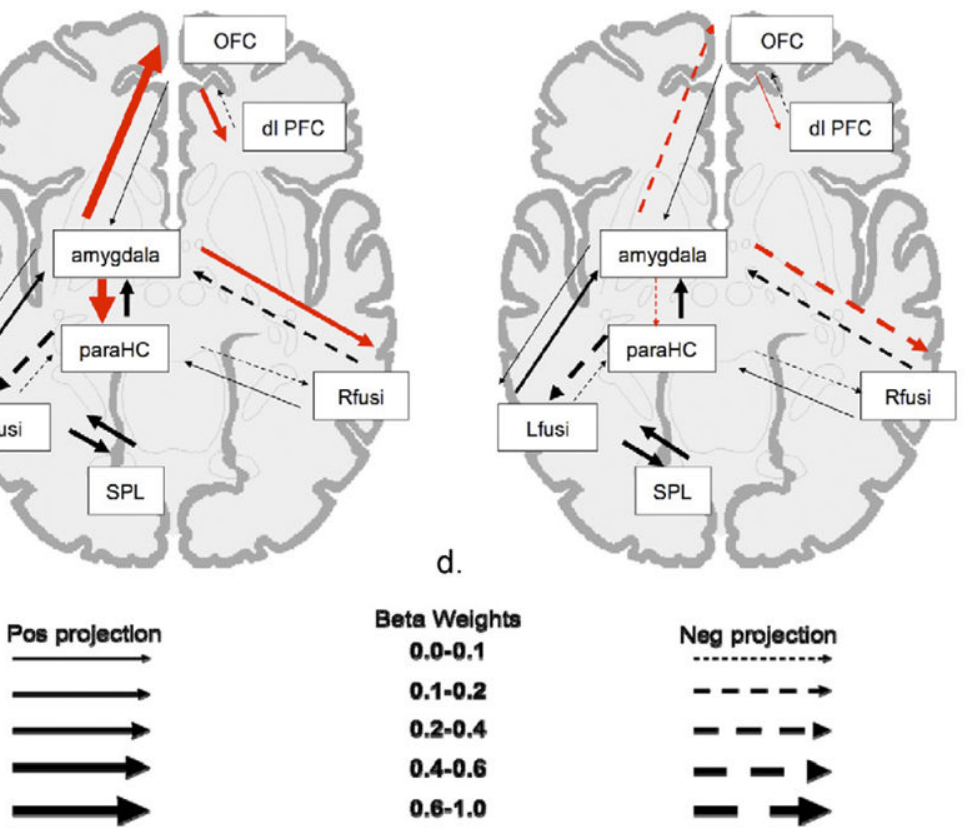

Fig. 4.

Model of encoding-related connectivity for selective emotional item memory. Visual depiction of the beta weights of path coefficients of functional networks related to remembering only the emotional item, while forgetting its background. As shown in legend, thicker arrows signify stronger connections between regions, solid arrows signify positive projections, and dashed arrows signify negative projections. Red arrows denote paths where coefficients for young and older adults significantly differ from each other. (a) Young adults positive scenes, (b) older adults positive scenes, (c) young adults negative scenes, (d) older adults negative scenes. Abbreviations: dlPFC, dorsolateral prefrontal cortex; Lfusi, left fusiform; OFC, orbitofrontal cortex; paraHC, parahippocampus; Rfusi, right fusiform; SPL, superior parietal lobule. 

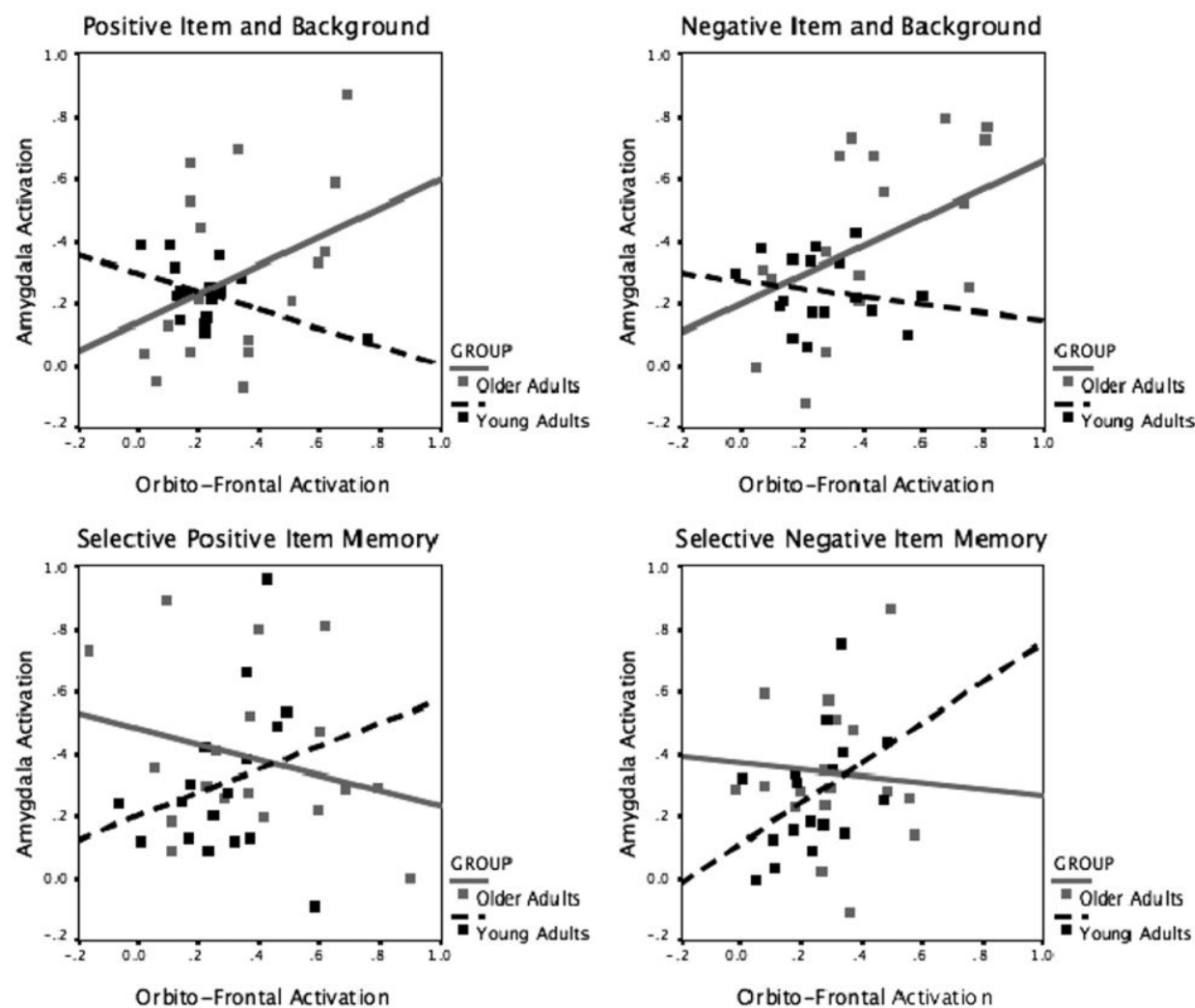

Fig. 5.

Correlations between levels of activity in the left amygdala and the right orbitofrontal cortex for young and older adults, by scene type and memory contents. 


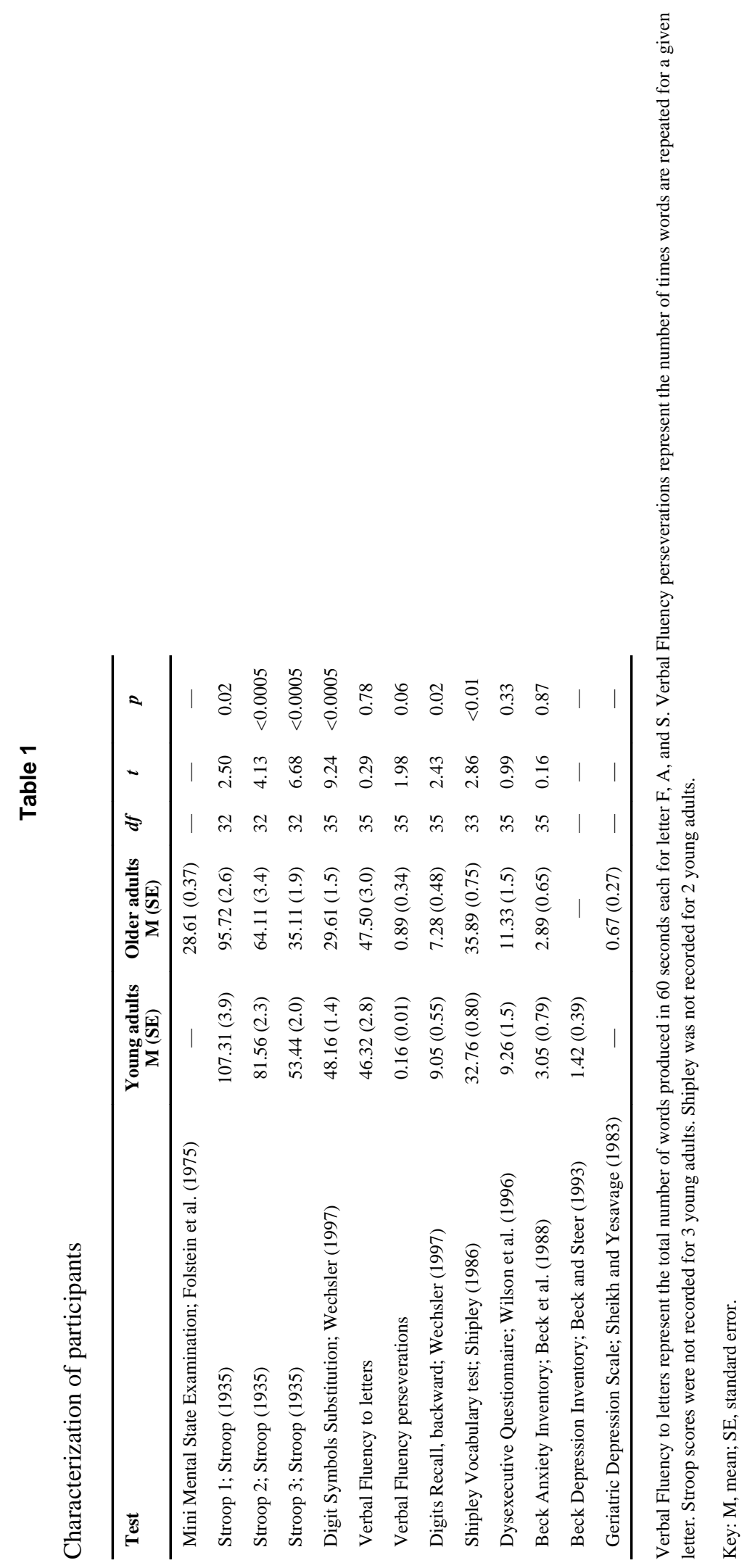

Neurobiol Aging. Author manuscript; available in PMC 2014 February 01. 
